\title{
LA FÓRMULA DE DESCOMPOSICIÓN PARA LA VALORACIÓN DE OPCIONES DE COMPRA BAJO EL MODELO DE HESTON.
}

\author{
RAÚL MERINO
}

\begin{abstract}
RESUMEn. En este artículo se presenta una breve introducción a los derivados financieros. Por una parte, recorremos parte de la historia y características de los derivados financieros. Por otra, se introduce el modelo de Black-Scholes-Merton, pilar básico de la valoración de opciones, y el modelo de Heston, uno de los modelos de volatilidad estocástica más populares. Además, se explica la teoría de la fórmula de descomposición que nos permite aproximar el precio de una opción en el modelo de Heston mediante el uso de la fórmula de Itô.
\end{abstract}

\section{INTRODUCCIÓN}

Es fácil pensar que los derivados financieros, y en general, cualquier producto financiero, es una invención de la economía moderna. Aunque más allá de la visión cinéfila de 'Wall Street' o los errores derivados de las crisis financieras; los derivados surgieron tan pronto como las personas fuimos capaces de hacer promesas creíbles. Los primeros derivados financieros sirvieron para garantizar el suministro de productos, facilitar el comercio y asegurar a los agricultores contra la perdida de cosechas. La primera evidencia de un contrato derivado se encuentra en la Ley 46 del código de Hammurabi, situado entre 1782 y 1750 a.C.

Para entender bien qué es un derivado financiero, hemos de dividirlos en dos categorías diferentes. Por un lado, tenemos la 'fuente primaria', a la que llamaremos 'subyacente', como pueden ser las acciones, bonos, materias primas, divisas, etc. Por otro lado, su contrato 'derivado', es un acuerdo en obtener un derecho en la entrega de un subyacente, o una cantidad monetaria dependiente de su evolución, en un momento futuro determinado.

Los contratos derivados más usuales son los futuros y las opciones. Un futuro es un acuerdo legal para comprar (o vender) un activo a un precio predeterminado en un momento especifico en el futuro. Mientras que una opción otorga el derecho, pero no la obligación, de comprar (o vender) un activo a un precio predeterminado en un momento especifico en el futuro. El precio acordado en el contrato se le llama 'Strike' y 'Moneyness' a la ratio entre el precio del activo respecto el Strike.

Una de las primeras historias relacionadas con la especulación de derivados se debe a Tales de Mileto. A fin de demostrar que los filósofos podrían hacerse ricos si ese fuese su objetivo, Tales utilizó sus conocimientos para predecir cómo sería la cosecha de aceitunas al verano siguiente. Al ver que sería abundante, decidió arrendar todas las prensas de oliva por adelantado. Como nadie sabía si la cosecha sería buena o mala, Tales compró los derechos a un precio relativamente bajo. Cuando la cosecha resultó ser abundante, la demanda de las prensas fue alta y Tales realquiló las prensas a precios mucho más elevados, obteniendo un beneficio considerable.

Pese a la antigüedad en el uso de contratos derivados, su modelización no comenzó hasta 1900. Louis Bachelier introdujo el primer modelo en su tesis 'Théorie de la spéculation', 5], siendo la piedra angular en la teoría de la valoración de derivados.

En su tesis, Bachelier se dio cuenta que había un equilibro entre compradores y vendedores.

2010 Mathematics Subject Classification. 60H05, 91G20, 91G30.

Palabras clave. Cálculo de opciones, modelos de volatilidad estocástica. 


\section{Parece que en el mercado, el conjunto de los especuladores, no puede creer ni en una subida ni en una caída del mercado, ya que, por cada precio cotizado, hay tantos compradores como vendedores.}

En particular, se dio cuenta de la necesidad de utilizar martingalas para describir los movimientos de los precios.

\section{La esperanza matemática de los especuladores es nula.}

Aunque el método para describir la evolución de los activos es similar a las metodologías actuales, Bachelier desarrolló su modelo bajo hipótesis de equilibrio, mientras que hoy en día se utilizan hipótesis de no arbitraje.

A pesar de las ideas novedosas utilizadas en la tesis de Bachelier, esta permaneció desconocida durante décadas. En 1953, Kendall, [26, sin ser consciente de la tesis de Bachelier, analizó 22 series de precios con el propósito de encontrar un modelo. En ese momento, se dio cuenta de que, entre los intervalos, había cambios aleatorios, descartando un efecto sistemático. Además, fue el primero en observar la dependencia temporal de la varianza empírica. Unos años más tarde, en 1959, Osborne, 34, encontró que los retornos logarítmicos del precio siguen un movimiento browniano.

A mediados de 1950, el estadista Jimmy Savage recuperó el trabajo de Bachelier y lo envió a diferentes amigos. Una de esas cartas llegó a Paul Samuelson, quien estaba trabajando en problemas relacionados con la valoración de opciones y warrants. Paul Samuelson se inspiró en el trabajo de Bachelier y relacionó el precio de las opciones con el uso de martingalas en 37 .

Unos años más tarde, en 1973, abrió la primera bolsa de opciones listadas, el CBOE, Chicago Board Options Exchange. Ese mismo año, se publicó el modelo de BlackScholes-Merton usando hipótesis de no-arbitraje, ver [9] y [30. Este modelo es la base de gran parte de los desarrollos acontecidos en años posteriores. El modelo de BlackScholes-Merton, o modelo BSM, proporciona una fórmula analítica que describe con parsimonia el precio de las opciones. La fórmula depende de la Moneyness, el tipo de interés y la volatilidad constante. La volatilidad constante es una de los principales inconvenientes del modelo. Pese a ello, se considera el modelo 'base' de mercado. Tanto es así, que el precio de las opciones cotizadas viene dado mediante la volatilidad de la fórmula de BSM. Estas volatilidades se les llama volatilidades implícitas, i.e. es la volatilidad constante que introducida en la fórmula de BSM obtiene el precio de mercado de la opción.

En los años siguientes, aparecieron nuevas versiones del modelo BSM intentando incluir ciertos patrones que se observaban en el mercado. El modelo CEV, Constant Elasticity Variance, fue presentado en un artículo sin publicar en [13, ver también 8. La principal mejora de este modelo es explicar la relación inversa entre el nivel del activo y la varianza de sus retornos. El siguiente año, Merton, 31, publicó el primer modelo de difusión con saltos. Este modelo extiende el modelo de BSM para procesos estocásticos con trayectorias no continuas, explicando posibles vibraciones 'anormales' debido a la llegada de nueva información con un efecto no marginal en el precio del activo. Los dos modelos introducen una asimetría en la estructura de volatilidades implícitas, también conocida como 'skew'. La asimetría significa que la volatilidad implícita decrece a medida que crece la Moneyness.

El 19 de octubre de 1987, también conocido como Lunes Negro, fue una de las mayores crisis financieras conocidas. Todos los mercados internacionales experimentaron grandes pérdidas, por ejemplo, el Dow Jones perdió en un día un $22.6 \%$ y se observó un aumento considerable de la volatilidad. Poco después del colapso, los traders observaron que las opciones cuyo Strike estaba alejado del valor actual del activo cotizaban inusualmente más caras comparadas con Strikes similares al valor del activo. Este fenómeno se llama 'la sonrisa de volatilidad'.

Una extensión natural del modelo de BSM es considerar que la volatilidad sigue un proceso estocástico. Este tipo de modelos se llaman modelos de volatilidad estocástica y surgieron con la finalidad de explicar mejor las superficies que veían los traders. 
Hubieron varios intentos para lograr obtener una solución, uno de los primeros fue 24], seguido de 25], 40] y [38] que intentaron resolver el problema mediante métodos numéricos. Usando un enfoque diferente, 23. obtuvo una aproximación del precio como una expansión de Taylor en el caso en que el activo y la varianza no estuvieran correlacionados. En [39] se propuso un modelo donde la volatilidad depende de un proceso de Ornstein-Ublenbeck aritmético no correlacionado con el activo. Mediante integración numérica es posible encontrar el precio de las opciones. En 1993 se publicó el modelo de Heston, 22, que se ha convertido en uno de los modelos más populares debido a que proporciona una solución semi-analítica y a sus buenas propiedades estadísticas. Heston propuso un modelo donde el proceso de varianza se modela mediante un proceso CIR, [14, y con una correlación arbitraria entre el activo y la varianza. Para un enfoque más detallado, ver [18 y las referencias que contiene.

Además de observar la 'sonrisa de volatilidad', las opciones que están cerca de su vencimiento se negocian con volatilidades implícitas más altas. Los traders, conscientes de la posibilidad de un gran movimiento de mercado, o salto en el precio de los activos, solicitan precios más altos. Los modelos de volatilidad estocástica no son capaces de reproducir toda la superficie de volatilidad cuando se ven volatilidades tan altas en los vencimientos cortos. Para mejorarlos, se introdujeron los modelos estocásticos de volatilidad con saltos. El primer modelo se le atribuye a [6], quien incorporó al modelo de Heston un proceso de saltos en el activo de manera similar a 31. Este modelo se ajusta mejor a la superficie de mercado. Existen diferentes alternativas utilizando amplitudes de salto diferentes, ver [41] o 27]. También es posible extender este modelo agregando saltos al proceso de varianza, por ejemplo, un modelo introducido por [16]. Sin embargo, según varios estudios empíricos, estos modelos tienden a sobreajustarse a los precios de mercado.

A pesar de la extensa literatura en los modelos de volatilidad estocástica, su uso es complejo. Por un lado, estos modelos necesitan calibrarse, es decir, se deben encontrar qué parámetros minimizan el error entre los precios del modelo y las opciones de mercado. Por otro lado, el cálculo es computacionalmente más intenso. [15, [17] y 36] propusieron un modelo diferente, el modelo de volatilidad local. Definieron una volatilidad instantánea única que es una función determinista del tiempo y el precio del activo consistente con los precios de las opciones de mercado.

Los modelos de volatilidad local son autoconsistentes, libres de arbitraje y se pueden calibrar con precisión para toda la superficie de volatilidad. Sin embargo, como señala 21], el comportamiento dinámico de la sonrisa y las asimetrías producidas por este modelo pueden ser contrarías al movimiento observado en el mercado, obteniendo peores coberturas que utilizando el modelo de BSM. En [21, se introduce el modelo SABR, este modelo se puede clasificar como un modelo de volatilidad local estocástico. Este modelo consiste en modelar el precio del activo con el modelo de CEV con un proceso de volatilidad estocástica exponencial. El principal éxito de este modelo es obtener un modelo capaz de ajustar la 'sonrisa de volatilidad' con pocos parámetros y con una aproximación sencilla de calcular de la volatilidad implícita.

La última tendencia en modelización consiste en considerar que la volatilidad sigue un proceso Volterra, en particular, se sustituye el browniano por un browniano fraccionario. Inicialmente, los pioneros de estos modelos, ver [10] y [11, supusieron que la volatilidad tenía memoria, es decir, era un proceso persistente con un parámetro de Hurst entre $H \in(1 / 2,1)$. En cambio, hoy en día, se están considerando los modelos antipersistentes, llamados modelos de volatilidad 'rough'. En estos modelos se observa una consistencia entre la serie temporal de la volatilidad realizada y la volatilidad fraccionaria aproximada. Esto significa que debería ser un modelo más consistente para valores opciones de mercado, ver, por ejemplo, [3], [7] y [19]. 


\section{Preliminares}

2.1. Derivados financieros. A menudo, las personas necesitan firmar acuerdos donde se comprometen a intercambiar diferentes activos o cantidades de dinero en momentos de tiempo diferentes. Por ejemplo, cuando alguien necesita comprar una casa, obtiene un préstamo hipotecario en el que recibe una gran suma de dinero a cambio de pagos mensuales futuros. Estos acuerdos son contratos financieros y a cada parte se le denomina contraparte.

Cuando un contrato financiero depende de la evolución de un activo, se le denomina contrato derivado. Esto se debe a que el precio del contrato se 'deriva' de la evolución del activo, también llamado subyacente. Nos referimos al precio del subyacente en el momento $t$ como $S_{t}$. Por ejemplo, un contrato derivado nos permitiría recibir una cantidad de efectivo hoy a cambio de $50 \mathrm{~kg}$ de arroz en el futuro. Este producto puede resultar interesante para agricultores, pudiendo adelantar parte de los ingresos de realizar la cosecha. Los derivados más simples son los futuros.

Definición 1. Un contrato de futuros es un acuerdo entre dos partes, donde una de ellas se compromete a comprar un activo a un precio específico a la otra, en un momento específico en el futuro. El momento en que se entrega el activo se denomina vencimiento y se indica con $T$. El precio especificado se conoce como $K$, también llamado precio de entrega. El valor del contrato a vencimiento es $S_{T}-K$ y su valor en el momento inicial es cero.

Un contrato de futuro es un juego de suma cero, una contraparte gana dinero y la otra lo pierde. Para valorar este contrato, no es necesario un modelo o el uso de probabilidades. Mediante hipótesis de no arbitraje se puede demostrar que un contrato de futuros es justo cuando el precio de entrega es el precio forward.

Definición 2. El precio forward de un activo con valor actual $S_{t}$ y vencimiento $T$ es

$$
F(t, T)=S_{t} e^{r(T-t)}
$$

donde $r$ es la tasa libre de riesgo.

No todos los contratos derivados pueden cotizarse sin el uso de un modelo. Todos aquellos donde una contraparte tiene la posibilidad de ejercitar o no el contrato, se debe especificar un modelo. Este tipo de productos se llaman opciones.

Definición 3. Una opción de compra europea es un acuerdo legal que otorga al tenedor el derecho, pero no la obligación, de comprar una unidad de un activo subyacente por un precio de ejercicio predeterminado $K$ en la fecha de vencimiento $T$. Si $S_{T}$ es el precio del activo subyacente en la fecha vencimiento $T$, entonces el valor, o pago, de una opción de compra es

$$
\left(S_{T}-K\right)_{+}= \begin{cases}S_{T}-K & \text { si } S_{T}>K, \\ 0 & \text { si } S_{T} \leq K .\end{cases}
$$

Si en el vencimiento, el precio del activo es mayor al precio acordado, el tenedor ejercerá la opción y obtendrá una ganancia. De lo contrario, como el tenedor obtendría una pérdida, este decidiría no ejercer la opción.

Definición 4. Una opción de venta europea es un acuerdo legal que otorga al tenedor el derecho, pero no la obligación, de vender una unidad de un activo subyacente por un precio de ejercicio predeterminado $K$ en la fecha de vencimiento $T$. Si $S_{T}$ es el precio del activo subyacente en la fecha vencimiento $T$, entonces el valor, o pago, de una opción de venta es

$$
\left(K-S_{T}\right)_{+}= \begin{cases}K-S_{T} & \text { si } S_{T}<K, \\ 0 & \text { si } S_{T} \geq K .\end{cases}
$$


Al contrario que los futuros, para adquirir una opción se debe pagar una prima. Este tipo de opciones se llaman plain vanilla y son las más sencillas. En inglés se utiliza el adjetivo plain vanilla para referirse a la versión más sencilla o común de algo, en analogía al sabor del helado de vainilla que se hizo ampliamente disponible y económico con el desarrollo de la vainilla artificial. Este tipo de opciones pueden ser europeas o americanas. Las opciones europeas sólo se pueden ejecutar en el vencimiento, mientras que las americanas se pueden ejecutar en cualquier momento anterior al vencimiento. Existen gran variedad de tipos de opciones con funciones de pago diferentes, a estas opciones se les conoce como opciones exóticas y se suelen contratar de manera privada entre dos contrapartes. Este tipo de contratación se llama OTC o Over-The-Counter.

Las opciones se clasifican dependiendo de cómo sea el precio de activo respecto de ejercicio:

Definición 5. Una opción está

- In the money o ITM si se ejecutará hoy, se obtendría un valor positivo. En el caso de una opción de compra si $S_{t}>K$.

- At the money o ATM si se ejecutará hoy, el valor del activo $S_{t}$ tiene el mismo nivel que el precio de ejercicio $K$. Es decir, si $S_{t}=K$.

- Out the money o OTM si se ejecutará hoy, el valor de la opción sería cero. En el caso de una opción de compra si $S_{t}<K$.

2.2. Breve introducción matemática. Para ser capaces de modelar la evolución de activos, es necesario introducir algunos elementos de cálculo estocástico. La teoría es extensa y, en este caso, introduciremos brevemente qué es un movimiento browniano, un proceso de Itô, la fórmula de Itô y la fórmula de Feynman-Kac.

Definición 6 (Movimiento browniano/Proceso de Wiener). Un movimiento browniano, o proceso de Wiener, es un proceso estocástico $\left(X_{t}\right)_{t \geq 0}$ tal que:

1. Las trayectorias $t \mapsto X_{t}$ son continuas con probabilidad 1 .

2. Para una secuencia finita de tiempo $t_{0}<t_{1}<\cdots<t_{n}$, los incrementos

$$
X_{t_{1}}-X_{t_{0}}, X_{t_{2}}-X_{t_{1}}, \cdots, X_{t_{n}}-X_{t_{n-1}}
$$

son independientes. Es decir, el comportamiento de un incremento no afecta al comportamiento de otros incrementos.

3. Tiene incrementos estacionarios. Es equivalente a decir que la distribución de los incrementos sólo depende de la longitud del incremento y no sobre el tiempo. Se puede escribir que para cualquier $t_{1}<t_{2}, X_{t_{2}}-X_{t_{1}} \sim X_{t_{2}-t_{1}}-X_{0}$.

La definición de movimiento browniano induce su propia distribución.

Teorema 7. Si $\left(X_{t}\right)_{t \geq 0}$ es un movimiento browniano, entonces $X_{t}-X_{0}$ es una variable aleatoria cuyos valores siguen una distribución normal con media rt y varianza $\sigma^{2} t$, donde $r$ y $\sigma$ son constantes positivas.

Demostración. Se puede ver la demostración en [12], Teorema 2.2.1.

Definición 8. Un movimiento browniano estándar es un movimiento browniano tal que $X_{0}=0$ c.s., $r=0$ y $\sigma^{2}=1$. Nos referimos al movimiento browniano estándar como $\left(W_{t}\right)_{t \geq 0}$.

Así como sabemos integrar respecto el tiempo, también estamos interesados en integrar respecto al movimiento browniano, es decir, ser capaces de resolver integrales estocásticas. Desafortunadamente, la definición de este tipo de integrales falla porque, aunque las trayectorias del movimiento browniano son continuas, no son de variación acotada ni diferenciables. Para poder definir este tipo de integrales, necesitamos que el integrando sea de cuadrado integrable y que sea adaptado a la información que genera el movimiento browniano. Este tipo de procesos se llaman adaptados. 
Cuando un proceso se escribe como la suma de una integral respecto el tiempo y otra integral respecto el movimiento browniano se llama procesos de Itô y son la base del cálculo estocástico.

Definición 9 (Proceso de Itô). Un proceso de Itô $\left(X_{t}\right)_{0 \leq t \leq T}$ es un proceso del tipo:

$$
X_{t}=X_{0}+\int_{0}^{t} K_{s} d s+\int_{0}^{t} H_{s} d W_{s},
$$

donde para todo $t \leq T$, tenemos que

1. $X_{0}$ es $\mathcal{F}_{0}$-medible.

2. $\left(K_{t}\right)$ y $\left(H_{t}\right)$ son procesos $\mathbb{F}$-adaptados.

3. $\int_{0}^{T}\left|K_{s}\right| d s<\infty \mathbb{P}$ c.s.

4. (Condición de cuadrado integrable) $\int_{0}^{T}\left|H_{s}\right|^{2} d s<\infty \mathbb{P}$ c.s.

Este tipo de procesos tienen la particularidad que tiene variación cuadrática diferente de cero. Es decir, si en análisis se considera que $(d t)^{2}=0$, en este caso $\left(d W_{t}\right)^{2}=d t$. Para poder trabajar con estos procesos, se necesita trabajar con una fórmula equivalente a la regla de la cadena, pero que funcione con los procesos de Itô. Esta fórmula es la fórmula de Itô y es uno de los teoremas fundamentales del cálculo estocástico.

Teorema 10 (Fórmula de Itô). Dado un proceso de Itô $\left(X_{t}\right)_{0 \leq t \leq T}$,

$$
X_{t}=X_{0}+\int_{0}^{t} K_{s} s+\int_{0}^{t} H_{s} d W_{s}
$$

y $f(t, x) \in \mathcal{C}^{1,2}$, entonces

$$
\begin{aligned}
f\left(t, X_{t}\right) & =f\left(0, X_{0}\right)+\int_{0}^{t} \partial_{s} f\left(s, X_{s}\right) d s+\int_{0}^{t} \partial_{x} f\left(s, X_{s}\right) d X_{s} \\
& +\frac{1}{2} \int_{0}^{t} \partial_{x}^{2} f\left(s, X_{s}\right) d[X, X]_{s}
\end{aligned}
$$

donde la variación cuadrática se define como

$$
[X, X]_{t}:=\int_{0}^{t} H_{s}^{2} d s
$$

Demostración. Se puede ver la demostración en [35, Capítulo II, Teorema 32.

La fórmula de Feynman-Kac nos permite expresar el precio de una opción como una solución de una ecuación diferencial en derivadas parciales. Es una consecuencia de la conexión entre las ecuaciones diferenciales estocásticas y ciertas ecuaciones diferenciales parciales parabólicas.

Teorema 11 (La Fórmula de Feynman-Kac). Sea $f(t, x)$ una función acotada, sea $\varphi(x)$ una función dos veces diferenciable, sea $V(t, x)$ una función acotada inferiormente para toda $x \in \mathbb{R}$. Dados unas funciones $\mu(t, x)$ y $\sigma^{2}(t, x)$ Lipschitz continua $y$ $u(t, x) \in \mathcal{C}^{1,2}$. Consideremos la ecuación diferencial en derivadas parciales

$$
\partial_{t} u(t, x)+\mu(t, x) \partial_{x} u(t, x)+\frac{1}{2} \sigma^{2}(t, x) \partial_{x}^{2} u(t, x)-V(t, x) u(t, x)+f(t, x)=0,
$$

definida para todo $x \in \mathbb{R}$ y $t \in[0, T]$, sujeta a la condición terminal

$$
u(x, T)=\varphi(x) .
$$

La solución de esta ecuación diferencial en derivadas parciales es la fórmula de Feynman-Kac y viene dada por la esperanza condicionada

$$
u(t, x)=\mathbb{E}\left[\int_{t}^{T} e^{-\int_{t}^{r} V\left(\tau, X_{\tau}\right) d \tau} f\left(r, X_{r}\right) d r+e^{-\int_{t}^{T} V\left(\tau, X_{\tau}\right) d \tau} \varphi\left(X_{T}\right) \mid X_{t}=x\right]
$$


sobre la medida de probabilidad subyacente tal que $X_{t}$ es un proceso de Itô definido por la ecuación

$$
d X_{t}=\mu(t, X) d t+\sigma(t, X) d W_{t},
$$

donde $W_{t}$ es un proceso de Wiener y la condición inicial de $X_{t}$ es $X_{t}=x$.

Demostración. Se puede ver la demostración en [33], Teorema 8.2.1.

\section{El modelo de Black-Scholes-Merton}

Consideremos que el vencimiento de una opción $T$ está fijado y $t \in[0, T]$. En un modelo financiero sencillo, existen dos tipos de activos: una cuenta bancaria $B_{t}$ que representa a un activo libre de riesgo y un activo con riesgo $S_{t}$. El precio de $B_{t}$ viene dado por

$$
B_{t}=e^{r t}
$$

donde $r \geq 0$ es la tasa libre de riesgo. Además, es la solución de una Ecuación Diferencial Ordinaria:

$$
d B_{t}=r B_{t} d t .
$$

El precio de un activo con riesgo $S_{t}$ viene dado por

$$
d S_{t}=\mu S_{t} d t+\sigma S_{t} d W_{t}
$$

donde $\mu$ es la tasa de crecimiento del activo, $\sigma>0$ es la volatilidad y $W_{t}$ es un movimiento browniano. Bajo argumentos de no arbitraje se puede ver que $\mu=r$.

Usando la fórmula de Itô, la ecuación diferencial estocástica (3) tiene la solución

$$
S_{t}=S_{0} e^{\left(r-\frac{1}{2} \sigma^{2}\right) t+\sigma W_{t}}
$$

donde $S_{0}>0$ es el valor actual del activo.

Este modelo tiene una solución analítica en el caso de opciones europeas.

TEOREMA 12. El precio de una opción de compra europea es

$$
C_{B S}\left(t, T, S_{t}, K, r, \sigma\right)=S_{t} \Phi\left(d_{+}\right)-K e^{-r \tau} \Phi\left(d_{-}\right)
$$

donde $S_{t}$ es el precio actual del subyacente, $\sigma$ es la volatilidad constante, $K$ es el precio de ejercicio, $\tau=T-t$ es el tiempo hasta vencimiento, $r$ es el tipo de interés libre de riesgo y $\Phi$ es la distribución acumulativa de una normal estándar. Los símbolos $d_{+} y$ $d_{-}$se refieren a las funciones siguientes

$$
d_{ \pm}=\frac{\ln \left(S_{t} / K\right)+\left(r \pm \frac{\sigma^{2}}{2}\right)(T-t)}{\sigma \sqrt{T-t}}
$$

Demostración. Queremos calcular cuál es el precio de una opción,

$$
C_{B S}\left(t, T, S_{t}, K, r, \sigma\right)=e^{-r(T-t)} \mathbb{E}\left[\left(S_{T}-K\right)_{+}\right] .
$$

El precio del activo subyacente en la fecha vencimiento, $S_{T}$, viene dado por

$$
S_{T}=S_{t} e^{\left(r-\frac{1}{2} \sigma^{2}\right)(T-t)+\sigma\left(W_{T}-W_{t}\right)} .
$$

Utilizando las propiedades del movimiento browniano, se puede escribir $W_{T}-W_{t}=$ $\sqrt{T-t} Z$ donde $Z$ es una distribución de probabilidad normal, es decir, $Z \sim \mathcal{N}(0,1)$. Podemos reescribir el precio de una opción cómo

$$
\begin{aligned}
C_{B S}\left(t, T, S_{t}, K, r, \sigma\right) & =e^{-r(T-t)} \mathbb{E}\left[\left(S_{t} e^{\left(r-\frac{1}{2} \sigma^{2}\right)(T-t)+\sigma \sqrt{T-t} Z}-K\right)_{+}\right] \\
& =e^{-r(T-t)} \int_{-\infty}^{\infty}\left(S_{t} e^{\left(r-\frac{1}{2} \sigma^{2}\right)(T-t)+\sigma \sqrt{T-t} z}-K\right)_{+} n(z) d z
\end{aligned}
$$


donde $n$ es la función de densidad de la normal. La integral es diferente de cero cuando $z \geq-d_{-}$, por lo tanto, se puede cambiar el dominio de la integral, obteniendo

$$
\begin{aligned}
C_{B S}(t, T, S, K, r, \sigma) & =e^{-r(T-t)} \int_{-d_{-}}^{\infty} \mathbb{E}\left[\left(S_{t} e^{\left(r-\frac{1}{2} \sigma^{2}\right)(T-t)+\sigma \sqrt{T-t} z}-K\right)_{+} n(z) d z\right] \\
& =S_{t} e^{-\frac{1}{2} \sigma^{2}(T-t)} \int_{-d_{-}}^{\infty} e^{\sigma \sqrt{T-t} z} n(z) d z-K e^{-r(T-t)} \int_{-d_{-}}^{\infty} n(z) d z \\
& =\frac{S_{t}}{\sqrt{2 \pi}} \int_{-d_{-}}^{\infty} e^{-\frac{1}{2}(z-\sigma(T-t))^{2}} d z-K e^{-r(T-t)} \Phi\left(d_{-}\right) \\
& =\frac{S_{t}}{\sqrt{2 \pi}} \int_{-d_{-}-\sigma \sqrt{T-t}}^{\infty} e^{-\frac{1}{2} u^{2}} d z-K e^{-r(T-t)} \Phi\left(d_{-}\right) \\
& =S_{t} \Phi\left(d_{+}\right)-K e^{-r \tau} \Phi\left(d_{-}\right) .
\end{aligned}
$$

Definición 13. Dada una opción europea cotizada en el mercado con precio $C$, strike $K$ y vencimiento $T$, la volatilidad implícita $I V=\sigma(K, T)$ es la volatilidad que utilizamos en la fórmula de BSM para obtener el precio de mercado de la opción. Es decir,

$$
C_{\text {Mercado }}=C_{B S}\left(0, T, S_{0}, K, r, \sigma(K, T)\right)
$$

donde $S_{0}$ es el precio del activo en el momento de negociación.

La solución para la volatilidad implícita es única debido a que la fórmula de BSM es monótonamente creciente en $\sigma$ con limites superiores o inferiores dependiendo de si es una opción de compra o de venta. Por el teorema de la función inversa, para cada precio hay una volatilidad implícita.
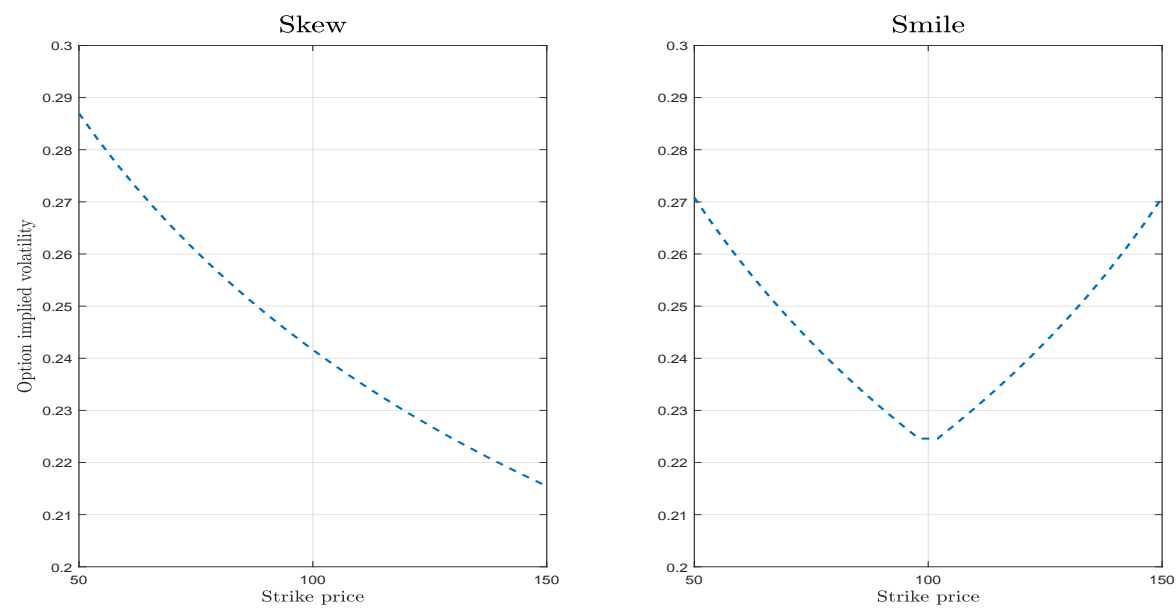

Figura 1. Ejemplo de Skew y Smile.

\section{El MODELO DE Heston}

El modelo de Heston, 22, es un modelo de volatilidad estocástica donde la varianza instantánea sigue un proceso de reversión a la media. La dinámica del modelo para el subyacente tiene en cuenta la asimetría y el exceso de curtosis observada en los retornos de los activos financieros además de ajustar los precios de mercado.

El proceso que modela el precio, $S$, sigue el siguiente sistema de ecuaciones diferenciales estocásticas

$$
\begin{aligned}
d S_{t} & =r S_{t} d t+\sigma_{t} S_{t}\left(\rho d W_{t}+\sqrt{1-\rho^{2}} d \widetilde{W}_{t}\right), \\
d \sigma_{t}^{2} & =\kappa\left(\theta-\sigma_{t}^{2}\right) d t+\nu \sqrt{\sigma_{t}^{2}} d W_{t} .
\end{aligned}
$$


El proceso $\sigma^{2}$ modela la varianza del precio, $\theta>0$ es el nivel medio de la varianza a largo plazo, $\kappa>0$ es la velocidad a la que la varianza vuelve a la media, $\nu>0$ es la volatilidad de la varianza y $r$ es la tasa de interés libre de riesgo. Las condiciones iniciales para el proceso de volatilidad $\sigma$ y el proceso de precio $S$ son $\sigma_{0}>0$ y $s_{0}>0$, respectivamente. Supondremos que se cumple la condición de Feller $2 \kappa \theta \geq \nu^{2}$ que evita que la varianza sea cero.

Heston expresó el precio de una opción de compra de manera similar a la fórmula de BSM, en su caso

$$
C_{\text {Heston }}=S P_{1}-K e^{-r(T-t)} P_{2}
$$

donde $P_{1}$ es la derivada respecto el valor del subyacente y $P_{2}$ es la probabilidad condicionada de que el activo sea más grande que el precio de ejercicio $K$ en la fecha de vencimiento. Las probabilidades $P_{1}$ y $P_{2}$ dependen de las funciones características $\varphi_{1}$ y $\varphi_{2}$ definidas mediante la inversa de la transformada de Fourier

$$
P_{j}=\frac{1}{2}+\frac{1}{\pi} \int_{0}^{\infty} \operatorname{Re}\left[\frac{e^{-i u \ln K} \varphi_{j}\left(S_{0}, \sigma_{0}^{2}, t, T, u\right)}{i u} d u\right] \quad j=1,2 .
$$

Heston asume que las funciones características $\varphi_{1} \mathrm{y} \varphi_{2}$ tiene la forma funcional

$$
\varphi_{j}(x, v, t, T, u)=\exp [C(\tau, \gamma)+D(\tau, \gamma) v+i \gamma x]
$$

donde

$$
\begin{aligned}
& C(\tau, \gamma)=r \gamma i \tau+\frac{\kappa \theta}{\nu^{2}}\left\{\left(b_{j}-\rho \nu \gamma i+d\right) \tau-2 \ln \left[\frac{1-g e^{d \tau}}{1-g}\right]\right\} \\
& D(\tau, \gamma)=\frac{b_{j}-\rho \nu \gamma i+d}{\nu^{2}}\left[\frac{1-e^{d \tau}}{1-g e^{d \tau}}\right]
\end{aligned}
$$

con

$$
\begin{array}{rlrl}
g & =\frac{b_{j}-\rho \nu \gamma i+d}{b_{j}-\rho \nu \gamma i-d}, \quad & d=\sqrt{\left(\rho \nu \gamma i-b_{j}\right)^{2}-\nu^{2}\left(2 u_{j} \gamma i-\gamma^{2}\right)} \\
u_{1} & =0,5, \quad u_{2}=-0,5, \quad a=\kappa \theta, \quad b_{1}=\kappa+\lambda-\rho \nu, \quad b_{2}=\kappa+\lambda
\end{array}
$$

Existen diversas mejoras a la fórmula propuesta por Heston, como puede ser the little Heston trap o la fórmula de Lewis. Se puede ver un resumen en [32].

\section{Fórmula de descomposición para el modelo de Heston.}

Existen diversos métodos para encontrar el valor de una opción para el modelo de Heston: desde fórmulas semi-analíticas hasta diferentes esquemas Monte Carlo. Además de estos métodos, se puede construir el precio de una opción a partir de una adaptación de la fórmula de BSM y la suma de términos extras que explican la componente estocástica de la volatilidad. Este método inspirado en [23] fue utilizado por primera vez en [1] donde se utilizan técnicas de Malliavin, más tarde en [2], 4] y [20] se usó utilizando el cálculo de Itô.

Consideramos $\mathcal{F}_{t}$ a la información disponible en el momento $t$, definimos la esperanza condicionada respecto la filtración natural $\left\{\mathcal{F}_{t}, t \geq 0\right\}$ de $S$ como $\mathbb{E}_{t}[\cdot]:=\mathbb{E}\left[\cdot \mid \mathcal{F}_{t}\right]$. Esta esperanza condicionada es el valor esperado en relación a la información disponible en el momento $t$. La esperanza condicionada de $\mathbb{E}_{t}[X]$ es la proyección de $X$ al espacios de los procesos adaptados, es decir, a los procesos definidos en $L^{2}$.

En el caso de un modelo de volatilidad no correlacionado, la fórmula para una opción de compra europea viene dado por

$$
V_{t}=E_{t}\left[B S\left(t, S_{t}, \bar{\sigma}_{t}\right)\right],
$$

donde $\bar{\sigma}^{2}(t)$ es la varianza media futura definida por

$$
\bar{\sigma}_{t}^{2}:=\frac{1}{T-t} \int_{t}^{T} \sigma_{s}^{2} d s .
$$


La igualdad (5) se llama la fórmula de Hull-White. Existen diferentes métodos para extender la fórmula para modelos correlacionados. La idea principal de 2 se basa en expandir el precio de una opción respecto a la proyección adaptada de la varianza media futura, es decir,

$$
v_{t}^{2}:=\mathbb{E}_{t}\left(\bar{\sigma}_{t}^{2}\right)=\frac{1}{T-t} \int_{t}^{T} \mathbb{E}_{t}\left[\sigma_{s}^{2}\right] d s
$$

Definimos

$$
M_{t}=\int_{0}^{T} \mathbb{E}_{t}\left[\sigma_{s}^{2}\right] d s
$$

Se puede reescribir la proyección adaptada de la varianza media futura como

$$
v_{t}^{2}:=\mathbb{E}_{t}\left(\bar{\sigma}_{t}^{2}\right)=\frac{1}{T-t}\left[M_{t}-\int_{0}^{t} \sigma_{s}^{2} d s\right]
$$

Entences, se obtiene que

$$
\begin{aligned}
d v_{t}^{2} & =\frac{d t}{(T-t)^{2}}\left[M_{t}-\int_{0}^{t} \sigma_{s}^{2} d s\right]+\frac{1}{T-t}\left[d M_{t}-\sigma_{t}^{2} d t\right] \\
& =\frac{1}{T-t}\left[d M_{t}+\left(v_{t}^{2}-\sigma_{t}^{2}\right) d t\right] .
\end{aligned}
$$

Sin pérdida de generalidad, podemos reescribir el modelo de precios usando el logaritmo del precio $X_{t}=\log \left(S_{t}\right)$ obteniendo

$$
d X_{t}=\left(r-\frac{1}{2} \sigma_{t}^{2}\right) d t+\sigma_{t}\left(\rho d W_{t}+\sqrt{1-\rho^{2}} d \widetilde{W}_{t}\right)
$$

Observación 1. A fin de generalizar la fórmula de descomposición, se asume que el vencimiento, $T$, el strike, $z$, y el tipo de interés, $r$, están fijados. Consideramos la función de pagos $A(t, T, x, z, r, y)=A(t, x, y)$. La función $A(t, x, y)$ pertenece al espacio $\mathcal{C}^{1,2,2}((0, T) \times(0, \infty) \times(0, \infty))$ donde $A$ es una vez diferenciable respecto a $t$ y dos veces diferenciable respecto a $x$ e $y$. Se asume que las derivadas son continuas.

A continuación, demostraremos cómo podemos descomponer el precio de una opción a partir de su valor terminal.

Teorema 14. Sea $X_{t}$ el proceso asociado al logaritmo del precio según (9), $\left\{B_{t}, t \in[0, T]\right\}$ una semimartingala continua respecto a la filtración $\mathcal{F}_{t}^{W}$ y sea el funcional $A(t, x, y)$ según la observación 1 . Para cada $t \in[0, T]$, tenemos la fórmula 
siguiente

$$
\begin{aligned}
\mathbb{E}_{t}\left[e^{-r(T-t)} A\left(T, X_{T}, v_{T}^{2}\right) B_{T}\right] & =A\left(t, X_{t}, v_{t}^{2}\right) B_{t} \\
+ & \mathbb{E}_{t}\left[\int_{t}^{T} e^{-r(u-t)} \partial_{y} A\left(u, X_{u}, v_{u}^{2}\right) B_{u} \frac{1}{T-u}\left(v_{u}^{2}-\sigma_{u}^{2}\right) d u\right] \\
+ & \mathbb{E}_{t}\left[\int_{t}^{T} e^{-r(u-t)} A\left(u, X_{u}, v_{u}^{2}\right) d B_{u}\right] \\
+ & \frac{1}{2} \mathbb{E}_{t}\left[\int_{t}^{T} e^{-r(u-t)}\left(\partial_{x}^{2}-\partial_{x}\right) A\left(u, X_{u}, v_{u}^{2}\right) B_{u}\left(\sigma_{u}^{2}-v_{u}^{2}\right) d u\right] \\
+ & \frac{1}{2} \mathbb{E}_{t}\left[\int_{t}^{T} e^{-r(u-t)} \partial_{y}^{2} A\left(u, X_{u}, v_{u}^{2}\right) B_{u} \frac{1}{(T-u)^{2}} d[M, M]_{u}\right] \\
+ & \rho \mathbb{E}_{t}\left[\int_{t}^{T} e^{-r(u-t)} \partial_{x, y}^{2} A\left(u, X_{u}, v_{u}^{2}\right) B_{u} \frac{\sigma_{u}}{T-u} d[W, M]_{u}\right] \\
+ & \rho \mathbb{E}_{t}\left[\int_{t}^{T} e^{-r(u-t)} \partial_{x} A\left(u, X_{u}, v_{u}^{2}\right) \sigma_{u} d[W, B]_{u}\right] \\
+ & \mathbb{E}_{t}\left[\int_{t}^{T} e^{-r(u-t)} \partial_{y} A\left(u, X_{u}, v_{u}^{2}\right) \frac{1}{T-u} d[M, B]_{u}\right] .
\end{aligned}
$$

Demostración. Si aplicamos la fórmula de Itô al proceso $e^{-r t} A\left(t, S_{t}, v_{t}^{2}\right) B_{t}$, se obtiene

$$
\begin{aligned}
& e^{-r T} A\left(T, X_{T}, v_{T}^{2}\right) B_{T}=e^{-r t} A_{B S}\left(t, X_{t}, v_{t}^{2}\right) B_{t} \\
& \text { - } r \int_{t}^{T} e^{-r u} A\left(u, X_{u}, v_{u}^{2}\right) B_{u} d u \\
& +\int_{t}^{T} e^{-r u} \partial_{u} A\left(u, X_{u}, v_{u}^{2}\right) B_{u} d u \\
& +\int_{t}^{T} e^{-r u} \partial_{x} A\left(u, X_{u}, v_{u}^{2}\right) B_{u} d X_{u} \\
& +\int_{t}^{T} e^{-r u} \partial_{y} A\left(u, X_{u}, v_{u}^{2}\right) B_{u} d v_{u}^{2} \\
& +\int_{t}^{T} e^{-r u} A\left(u, X_{u}, v_{u}^{2}\right) d B_{u} \\
& +\frac{1}{2} \int_{t}^{T} e^{-r u} \partial_{x}^{2} A\left(u, X_{u}, v_{u}^{2}\right) B_{u} d[X, X]_{u} \\
& +\frac{1}{2} \int_{t}^{T} e^{-r u} \partial_{y}^{2} A\left(u, X_{u}, v_{u}^{2}\right) B_{u} d\left[v^{2}, v^{2}\right]_{u} \\
& +\int_{t}^{T} e^{-r u} \partial_{x, y}^{2} A\left(u, X_{u}, v_{u}^{2}\right) B_{u} d\left[X, v^{2}\right]_{u} \\
& +\int_{t}^{T} e^{-r u} \partial_{x} A\left(u, X_{u}, v_{u}^{2}\right) d[X, B]_{u} \\
& +\int_{t}^{T} e^{-r u} \partial_{y} A\left(u, X_{u}, v_{u}^{2}\right) d\left[v^{2}, B\right]_{u} .
\end{aligned}
$$


Desarrollando, obtenemos

$$
\begin{aligned}
& e^{-r T} A\left(T, X_{T}, v_{T}^{2}\right) B_{T}=e^{-r t} A\left(t, X_{t}, v_{t}^{2}\right) B_{t} \\
& \text { - } r \int_{t}^{T} e^{-r u} A\left(u, X_{u}, v_{u}^{2}\right) B_{u} d u \\
& +\int_{t}^{T} e^{-r u} \partial_{u} A\left(u, X_{u}, v_{u}^{2}\right) B_{u} d u \\
& +\int_{t}^{T} e^{-r u} \partial_{x} A\left(u, X_{u}, v_{u}^{2}\right) B_{u}\left(r-\frac{1}{2} \sigma_{u}^{2}\right) d u \\
& +\rho \int_{t}^{T} e^{-r u} \partial_{x} A\left(u, X_{u}, v_{u}^{2}\right) B_{u} \sigma_{u} d W_{u} \\
& +\sqrt{1-\rho^{2}} \int_{t}^{T} e^{-r u} \partial_{x} A\left(u, X_{u}, v_{u}^{2}\right) B_{u} \sigma_{u} d \widetilde{W}_{u} \\
& +\int_{t}^{T} e^{-r u} \partial_{y} A\left(u, X_{u}, v_{u}^{2}\right) \frac{B_{u}}{T-u} d M_{u} \\
& +\int_{t}^{T} e^{-r u} \partial_{y} A\left(u, X_{u}, v_{u}^{2}\right) B_{u} \frac{v_{u}^{2}-\sigma_{u}^{2}}{T-u} d u \\
& +\int_{t}^{T} e^{-r u} A\left(u, X_{u}, v_{u}^{2}\right) d B_{u} \\
& +\frac{1}{2} \int_{t}^{T} e^{-r u} \partial_{x}^{2} A\left(u, X_{u}, v_{u}^{2}\right) B_{u} \sigma_{u}^{2} d u \\
& +\frac{1}{2} \int_{t}^{T} e^{-r u} \partial_{y}^{2} A\left(u, X_{u}, v_{u}^{2}\right) \frac{B_{u}}{(T-u)^{2}} d[M, M]_{u} \\
& +\rho \int_{t}^{T} e^{-r u} \partial_{x, y}^{2} A\left(u, X_{u}, v_{u}^{2}\right) \frac{B_{u} \sigma_{u}}{T-u} d[W, M]_{u} \\
& +\sqrt{1-\rho^{2}} \int_{t}^{T} e^{-r u} \partial_{x, y}^{2} A\left(u, X_{u}, v_{u}^{2}\right) \frac{B_{u} \sigma_{u}}{T-u} d[\widetilde{W}, M]_{u} \\
& +\rho \int_{t}^{T} e^{-r u} \partial_{x} A\left(u, X_{u}, v_{u}^{2}\right) \sigma_{u} d[W, B]_{u} \\
& +\sqrt{1-\rho^{2}} \int_{t}^{T} e^{-r u} \partial_{x} A\left(u, X_{u}, v_{u}^{2}\right) \sigma_{u} d[\widetilde{W}, B]_{u} \\
& +\int_{t}^{T} e^{-r u} \partial_{y} A\left(u, X_{u}, v_{u}^{2}\right) \frac{1}{T-u} d[M, B]_{u} .
\end{aligned}
$$

Añadiendo y quitando el termino

$$
\frac{1}{2} \int_{t}^{T} e^{-r u} \partial_{x}^{2} A\left(u, X_{u}, v_{u}^{2}\right) B_{u} v_{u}^{2} d u
$$


tenemos que

$$
\begin{aligned}
& e^{-r T} A\left(T, X_{T}, v_{T}^{2}\right) B_{T}=e^{-r t} A\left(t, X_{t}, v_{t}^{2}\right) B_{t} \\
& \text { - } r \int_{t}^{T} e^{-r u} A\left(u, X_{u}, v_{u}^{2}\right) B_{u} d u \\
& +\int_{t}^{T} e^{-r u} \partial_{u} A\left(u, X_{u}, v_{u}^{2}\right) B_{u} d u \\
& +\int_{t}^{T} e^{-r u} \partial_{x} A\left(u, X_{u}, v_{u}^{2}\right) B_{u}\left(r-\frac{1}{2} \sigma_{u}^{2}\right) d u \\
& +\rho \int_{t}^{T} e^{-r u} \partial_{x} A\left(u, X_{u}, v_{u}^{2}\right) B_{u} \sigma_{u} d W_{u} \\
& +\sqrt{1-\rho^{2}} \int_{t}^{T} e^{-r u} \partial_{x} A\left(u, X_{u}, v_{u}^{2}\right) B_{u} \sigma_{u} d \widetilde{W}_{u} \\
& +\int_{t}^{T} e^{-r u} \partial_{y} A\left(u, X_{u}, v_{u}^{2}\right) \frac{B_{u}}{T-u} d M_{u} \\
& +\int_{t}^{T} e^{-r u} \partial_{y} A\left(u, X_{u}, v_{u}^{2}\right) B_{u} \frac{v_{u}^{2}-\sigma_{u}^{2}}{T-u} d u \\
& +\int_{t}^{T} e^{-r u} A\left(u, X_{u}, v_{u}^{2}\right) d B_{u} \\
& +\frac{1}{2} \int_{t}^{T} e^{-r u} \partial_{x}^{2} A\left(u, X_{u}, v_{u}^{2}\right) B_{u} \sigma_{u}^{2} d u \\
& +\frac{1}{2} \int_{t}^{T} e^{-r u} \partial_{y}^{2} A\left(u, X_{u}, v_{u}^{2}\right) \frac{B_{u}}{(T-u)^{2}} d[M, M]_{u} \\
& +\rho \int_{t}^{T} e^{-r u} \partial_{x, y}^{2} A\left(u, X_{u}, v_{u}^{2}\right) B_{u} \frac{\sigma_{u}}{T-u} d[W, M]_{u} \\
& +\rho \int_{t}^{T} e^{-r u} \partial_{x} A\left(u, X_{u}, v_{u}^{2}\right) \sigma_{u} d[W, B]_{u} \\
& +\int_{t}^{T} e^{-r u} \partial_{y} A\left(u, X_{u}, v_{u}^{2}\right) \frac{1}{T-u} d[M, B]_{u} \\
& +\frac{1}{2} \int_{t}^{T} e^{-r u} \partial_{x}^{2} A\left(u, X_{u}, v_{u}^{2}\right) B_{u} v_{u}^{2} d u \\
& -\frac{1}{2} \int_{t}^{T} e^{-r u} \partial_{x}^{2} A\left(u, X_{u}, v_{u}^{2}\right) B_{u} v_{u}^{2} d u .
\end{aligned}
$$

Si agrupamos los términos que están en recuadros, tenemos la fórmula de FeynmanKac asociada al proceso (9), estos términos se anulan. Multiplicando por $e^{-r t}$ y usando esperanzas condicionadas la demostración finaliza.

A fin de abreviar la formulación, definimos los operadores siguientes respecto al logaritmo del precio:

- $\Lambda:=\partial_{x}$,

- $\Gamma:=\left(\partial_{x}^{2}-\partial_{x}\right)$,

- $\Gamma^{2}=\Gamma \circ \Gamma$.

El siguiente corolario nos servirá de base para descomponer en el precio de una opción, así como también obtener una aproximación. 
Corolario 15. Sea la función A y el proceso B como en el Teorema 14. Supongamos que la función A cumple

$$
\partial_{y} A(t, x, y)=\frac{(T-t)}{2}\left(\partial_{x}^{2}-\partial_{x}\right) A(t, x, y) .
$$

Consideremos que $A_{t}:=A\left(t, X_{t}, v_{t}^{2}\right) \forall t \in[0, T]$. Para todo $t \in[0, T]$, tenemos que

$$
\begin{aligned}
e^{-r(T-t)} E_{t}\left[A_{T} B_{T}\right] & =A_{t} B_{t} \\
& +\frac{\rho}{2} E_{t}\left[\int_{t}^{T} e^{-r(u-t)} \Lambda \Gamma A_{u} B_{u} \sigma_{u} d[W, M]_{u}\right] \\
& +\frac{1}{8} E_{t}\left[\int_{t}^{T} e^{-r(u-t)} \Gamma^{2} A_{u} B_{u} d[M, M]_{u}\right] \\
& +\rho E_{t}\left[\int_{t}^{T} e^{-r(u-t)} \Lambda A_{u} \sigma_{u} d[W, B]_{u}\right] \\
& +\frac{1}{2} E_{t}\left[\int_{t}^{T} e^{-r(u-t)} \Gamma A_{u} d[M, B]_{u}\right] \\
& +E_{t}\left[\int_{t}^{T} e^{-r(u-t)} A_{u} d B_{u}\right] .
\end{aligned}
$$

Demostración. Substituyendo 10 en el Teorema 14 y usando las definiciones de $\Lambda$ y $\Gamma$ se completa la prueba.

Ahora ya tenemos todos los elementos para poder aplicar la fórmula de descomposición a una opción de compra (o de venta). Realicemos el cambio de variable

$$
C_{\widetilde{B S}}(t, x, y):=C_{B S}\left(t, e^{x}, \sqrt{y}\right) .
$$

Consideremos que $C_{S V}(t, x, y)$ es el precio de una opción de compra respecto un modelo de volatilidad estocástica. Como hemos hecho anteriormente, podemos hacer un cambio de variables

$$
C_{\widetilde{S V}}(t, x, y):=C_{S V}\left(t, e^{x}, \sqrt{y}\right) .
$$

En el vencimiento, todas las fórmulas valen lo mismo, el valor del subyacente es conocido, es decir:

$$
V_{T}=C_{S V}\left(T, S_{T}, \sigma_{T}\right)=C_{\widetilde{S V}}\left(T, X_{T}, v_{T}^{2}\right)=C_{\widetilde{B S}}\left(T, X_{T}, v_{T}^{2}\right) .
$$

Si aplicamos el Corolario 15 con $A=C_{\overparen{B S}}$ y $B \equiv 1$, se puede descomponer el precio de una opción de compra en un modelo de volatilidad estocástica, es decir $C_{S V}\left(t, S_{t}, \sigma\right)$, con la fórmula:

$$
\begin{aligned}
V_{t} & =C_{B S}\left(t, S_{t}, v_{t}\right) \\
& +\frac{1}{8} \mathbb{E}_{t}\left[\int_{t}^{T} e^{-r(u-t)} \Gamma^{2} C_{\overparen{B S}}\left(u, X_{u}, v_{u}^{2}\right) d[M, M]_{u}\right] \\
& +\frac{\rho}{2} \mathbb{E}_{t}\left[\int_{t}^{T} e^{-r(u-t)} \Lambda \Gamma C_{\overparen{B S}}\left(u, X_{u}, v_{u}^{2}\right) \sigma_{u} d[W, M]_{u}\right] \\
& =C_{B S}\left(t, S_{t}, v_{t}\right)+(I)+(I I) .
\end{aligned}
$$

Si miramos la demostración con cierta calma, se puede ver que no hemos utilizado casi ninguna hipótesis sobre la volatilidad, exceptuando que sea positiva y que la solución de la ecuación diferencial estocástica exista y sea única. Por lo tanto, esta descomposición funciona para cualquier modelo de volatilidad estocástica. Desgraciadamente es una solución complicada de calcular numéricamente. 
Si aplicamos el Corolario 10 a los términos (I) y (II), se puede conseguir una aproximación del modelo de volatilidad que es equivalente a 'congelar' la derivada en el momento inicial. En este caso, obtendríamos

$$
\begin{aligned}
V_{t} & =C_{B S}\left(t, S_{t}, v_{t}\right) \\
& +\frac{1}{8} \Gamma^{2} C_{\widetilde{B S}}\left(t, X_{t}, v_{t}^{2}\right) \mathbb{E}_{t}\left[\int_{t}^{T} d[M, M]_{u}\right] \\
& +\frac{\rho}{2} \Lambda \Gamma C_{\widetilde{B S}}\left(t, X_{t}, v_{t}^{2}\right) \mathbb{E}_{t}\left[\int_{t}^{T} \sigma_{u} d[W, M]_{u}\right] \\
& +\epsilon_{t} .
\end{aligned}
$$

$\mathrm{Al}$ aplicar el corolario, aparecen nuevos términos que son descartados y considerados como el error $\epsilon_{t}$ de la aproximación. Este error se puede acotar en función del modelo de volatilidad estocástica seleccionado. En [4], se obtiene que el error en el modelo de Heston se puede acotar por

$$
\left|\epsilon_{t}\right| \leq\left(\nu^{2}(|\rho|+\nu)^{2}\right)\left(\frac{1}{r} \wedge(T-t)\right) \Pi(\kappa, \theta),
$$

donde $\Pi(\kappa, \theta)$ es una constante positiva que depende de $\kappa$ y $\theta$.
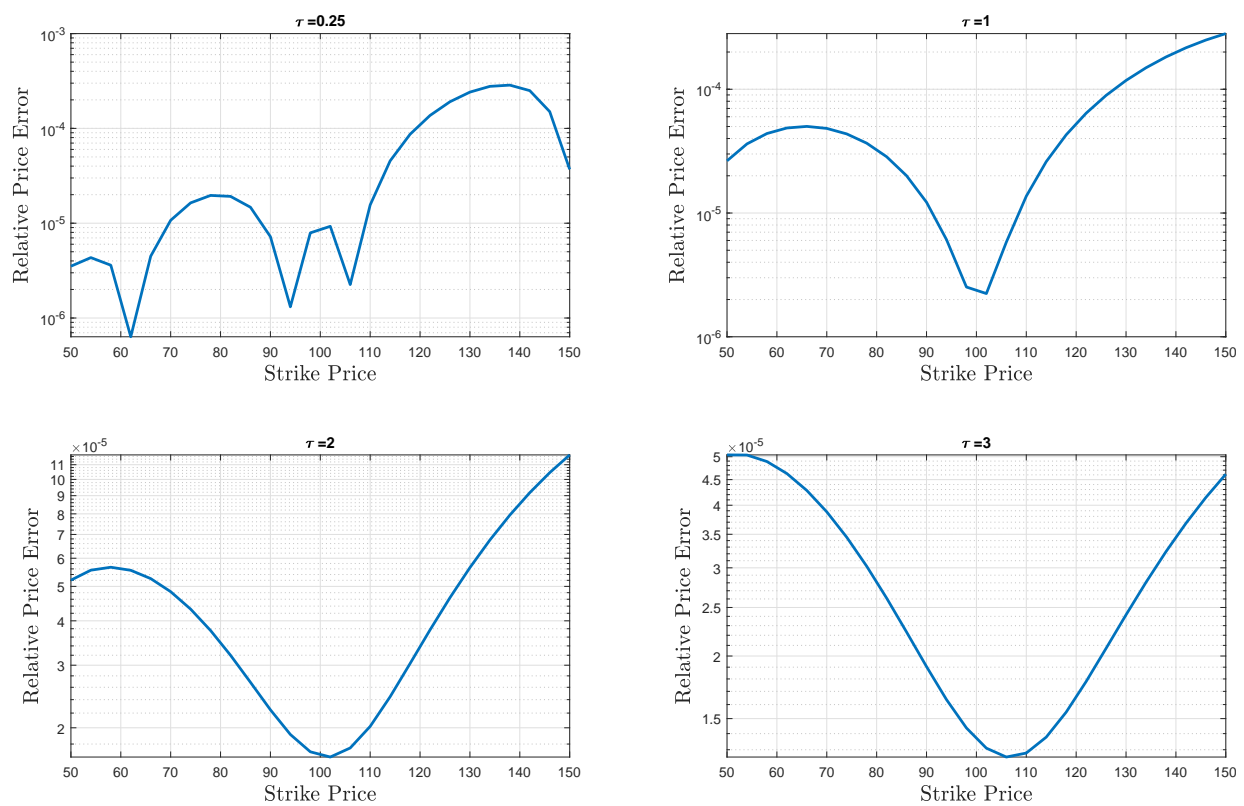

Figura 2. Error de la fórmula de descomposición para diferentes vencimientos.

Es posible mejorar la aproximación, sólo es necesario aplicar el Corolario 15 a cada nuevo término. Se pueden seleccionar qué términos aproximar para conseguir el orden deseado. En [20] se proponen nuevas fórmulas de aproximación, así como una comparativa con otros métodos existentes en la literatura.

Esta técnica nos permite expresar el precio de la opción en un modelo de volatilidad estocástica como la fórmula de BSM, respecto la proyección de la volatilidad futura, y una corrección que depende de sus derivadas. Además, es bastante genérica y se puede utilizar para otros modelos. Por ejemplo, para el modelo de Bates como en [28] o para un modelo de volatilidad local como el CEV, ver [29]. 


\section{Conclusiones}

Sin lugar a duda, el siglo XX ha sido el siglo de oro de los derivados financieros. En este artículo, se explica una breve historia de los derivados financieros con las referencias clásicas de la literatura así como las nuevas tendencias. Además de introducir las definiciones básicas de las opciones de compra, se explica el modelo de Black-ScholesMerton que es uno de los pilares básicos de la valoración de activos, siendo la base de modelos más sofisticados. Se introduce también el modelo de Heston, uno de los modelos de volatilidad estocástica más populares con su solución original. Adicionalmente, se presenta la teoría de la fórmula de descomposición, la cual nos permite aproximar el precio de una opción mediante la fórmula Black-Scholes-Merton, respecto la proyección de la volatilidad futura, y unos términos adicionales que explican la componente de la volatilidad estocástica. Se observa la calidad numérica del ajuste en varios casos.

AGRADECIMIENTos. El autor expresa su gratitud a Jorge A. León y al árbitro anónimo por sus comentarios y sugerencias a fin de mejorar la calidad del artículo.

\section{REFERENCIAS}

[1] Alòs E., A generalization of the Hull and White formula with applications to option pricing approximation, Finance and Stochastics, (2006).

[2] Alòs E., A decomposition formula for option prices in the Heston model and applications to option pricing approximation, Finance and Stochastics, (2012).

[3] Alòs E., León J. A., Vives J., On the short-time behavior of the implied volatility for jumpdiffusion models with stochastic volatility, Finance and Stochastics, (2007).

[4] Alòs E., de Santiago R., Vives J., Calibration of stochastic volatility models via second-order approximation: The Heston case, International Journal of Theoretical and Applied Finance, (2015).

[5] Bachelier L., Théorie de la spéculation, PhD thesis, Paris: Gauthier-Villars, 1900.

[6] Bates D. S., Jumps and stochastic volatility: Exchange rate processes implicit in Deutsche mark options, Review of Financial Studies, (1996).

[7] Bayer C., Friz P., Gatheral J., Pricing under rough volatility, Quantitative Finance, (2016).

[8] Beckers S., The constant elasticity of variance model and its implications for option pricing, The Journal of Finance, (1980).

[9] Black F. S., Scholes M. S., The pricing of options and corporate liabilities, Journal of Political Economy, (1973).

[10] Comte F., Renault E., Long memory in continuous-time stochastic volatility models, Mathematical Finance, An International Journal of Mathematics, Statistics and Financial Economics, (1998).

[11] Comte F., Coutin L., Renault E., Affine fractional stochastic volatility models, Annals of Finance, (2012).

[12] Corcuera J. M., Quantitative finance, (2018).

[13] Cox J. C., Notes on option pricing I: Constant elasticity of variance diffusions, The Journal of Portfolio Management, (1975).

[14] Cox J. C., Ingersoll J. E., Ross S. A., A theory of the term structure of interest rates, Econometrica. Journal of the Econometric Society, (1985).

[15] Derman E., Kani I., Riding on a smile, Risk, (1994).

[16] Duffie D., Pan J., Singleton K., Transform analysis and asset pricing for affine jump-diffusions, Econometrica, Journal of the Econometric Society, (2000).

[17] Dupire B., Pricing with a smile, Risk, (1994).

[18] Fouque J-P., Papanicolaou G., Sircar K. R., Derivatives in financial markets with stochastic volatility, Cambridge University Press, Cambridge, U.K., (2000).

[19] Gatheral J., Jaisson T., Rosenbaum M., Volatility is rough, Quantitative Finance, (2018).

[20] Gulisashvili A., Lagunas M., Merino R., Vives J., Higher order approximation of call option prices under stochastic volatility models, Journal of Computational Finance, (2020).

[21] Hagan P. S., Kumar D., Lesniewski A., Woodward D. E., Managing smile risk, Wilmott Magazine, (2002).

[22] Heston S. L., A closed-form solution for options with stochastic volatility with applications to bond and currency options, The Review of Financial Studies, (1993).

[23] Hull J. C., White A. D., The pricing of options on assets with stochastic volatilities, Journal of Finance, (1987).

[24] Johnson H. E., Option pricing when the variance is changing, Graduate School of Management, (1979). 
[25] Johnson H. E., Shanno D., Option pricing when the variance is changing, Graduate School of Administration, (1985).

[26] Kendall M.G., The analysis of economic time-series part I: Prices, Journal of the Royal Statistical Society, Series A (General), (1953).

[27] Kou S. G., A jump-diffusion model for option pricing, Management Science, (2002).

[28] Mrázek M., Pospíšil J., Sobotka T., Vives J., Decomposition formula for jump diffusion models, International Journal of Theoretical and Applied Finance, (2018).

[29] Merino R., Vives J., Option price decomposition in spot-dependent volatility models and some applications, International Journal of Stochastic Analysis, (2017).

[30] Merton R. C., Theory of rational option pricing, Economics and Management Science, (1973).

[31] Merton R. C., Option pricing when underlying stock returns are discontinuous, Journal of Financial Economics, (1976).

[32] Mrázek M., Pospíšil J., Calibration and simulation of Heston model, Open Mathematics, (2017).

[33] Øksendal B., Stochastic differential equations: An introduction with applications, Universitext, Springer-Verlag, Berlin, (2003).

[34] Osborne MFM., Brownian motion in the stock market, Operations Research, (1959).

[35] Protter P., Stochastic integration and differential equations, Springer-Verlag Berlin Heidelberg, (2004).

[36] Rubinstein M., Implied binomial trees, Journal of Finance, (1994).

[37] Samuelson P., Rational theory of warrant pricing, Industrial Management Review, (1965).

[38] Scott L. O., Option pricing when the variance changes randomly: Theory, estimation, and an application, The Journal of Financial and Quantitative Analysis, (1987).

[39] Stein J., Stein E., Stock price distributions with stochastic volatility: An analytic approach, Review of Financial Studies, (1991).

[40] Wiggins J. B., Option values under stochastic volatility: Theory and empirical estimates, Journal of Financial Economics, (1987).

[41] Yan G., Hanson F. B., Option pricing for a stochastic-volatility jump-diffusion model with loguniform jump-amplitude, Proceedings of American Control Conference, IEEE, (2006).

Dirección del autor:

VidaCaixa S.A.,

Unidad de Gestión de Riesgo de Mercado,

C/Juan Gris, 2-8, 08014 Barcelona, Spain.

e-mail: raul .merino85@gmail.com 